\title{
Adaptation to Temporal Contrast in Primate and Salamander Retina
}

\author{
Divya Chander and E. J. Chichilnisky \\ Systems Neurobiology, The Salk Institute, La Jolla, California 92037-1099
}

\begin{abstract}
Visual adaptation to temporal contrast (intensity modulation of a spatially uniform, randomly flickering stimulus) was examined in simultaneously recorded ensembles of retinal ganglion cells (RGCs) in tiger salamander and macaque monkey retina. Slow contrast adaptation similar to that recently discovered in salamander and rabbit retina was observed in monkey retina. $A$ novel method was developed to quantify the effect of temporal contrast on steady-state sensitivity and kinetics of light responses, separately from nonlinearities that would otherwise significantly contaminate estimates of sensitivity. Increases in stimulus contrast progressively and reversibly attenuated and sped light responses in both salamander and monkey RGCs, indicating that a portion of the contrast adaptation observed in visual cortex originates in the retina. The effect of adaptation on
\end{abstract}

sensitivity and kinetics differed in simultaneously recorded populations of ON and OFF cells. In salamander, adaptation affected the sensitivity of OFF cells more than ON cells. In monkey, adaptation affected the sensitivity of ON cells more than OFF cells. In both species, adaptation sped the light responses of OFF cells more than ON cells. Functionally defined subclasses of ON and OFF cells also exhibited asymmetric adaptation. These findings indicate that contrast adaptation differs in parallel retinal circuits that convey distinct visual signals to the brain.

Key words: retinal ganglion cell; salamander; primate; retina; contrast; temporal contrast; adaptation; gain; sensitivity; nonlinear; white noise
Visual processing in the retina adjusts dynamically, or adapts, to accommodate changes in the viewing environment. For example, a sustained increase in mean light level reduces behavioral sensitivity and attenuates light responses in retinal ganglion cells (RGCs) via mechanisms at several sites in the retinal circuitry (Shapley and Enroth-Cugell, 1984). By dynamically controlling sensitivity, adaptation allocates the finite range of neural signals to the range of intensities expected from recent experience. This may improve the efficiency of visual coding and maximize stimulus discriminability.

Several studies have also indicated that a sustained change in stimulus temporal contrast (amplitude of intensity variations about the mean) alters behavioral sensitivity (Blakemore and Campbell, 1969; Lorenceau, 1987; Schieting and Spillmann, 1987; Greenlee et al., 1991; Anstis, 1996) and light responses in RGCs. A fast form of adaptation was inferred from differences in RGC response amplitude and kinetics with low and high contrast stimuli (Shapley and Victor, 1978; 1981; Victor, 1987; Benardete et al., 1992). This phenomenon, dubbed contrast gain control, was modeled as a feedback nonlinearity that occurs within tens of milliseconds of stimulus onset (Shapley and Victor, 1981; Victor, 1987). A slower form of adaptation was observed after a step in stimulus contrast: firing rate changed abruptly and then settled to a new level over tens of seconds (Smirnakis et al., 1997). More

Received June 12, 2001; revised Sept. 7, 2001; accepted Sept. 17, 2001.

This work was supported by National Institutes of Health Grant EY-13150, the Alfred P. Sloan Foundation, and the McKnight Foundation (E.J.C.); and the Chapman Charitable Trust, the Legler-Benbough Foundation, and MSTP Training Grant GM07198 (D.C.). We thank E. Callaway, S. Zola, and T. Albright for providing access to tissue; F. Rieke and K. Kim for critical input and comments on this manuscript; G. Horwitz, G. Stoner, M. Feller, and S. du Lac for comments on this manuscript; R. Kalmar for assistance during experiments; and S. Barry and R. Roder for technical assistance.

Correspondence should be addressed to Dr. E. J. Chichilnisky, Systems Neurobiology, The Salk Institute, 10010 North Torrey Pines Road, La Jolla, CA 920371099. E-mail: ej@salk.edu.

Copyright (ㄷ) 2001 Society for Neuroscience $\quad 0270-6474 / 01 / 219904-13 \$ 15.00 / 0$ recent findings indicate that contrast adaptation consists of several temporally distinct components (Brown and Masland, 2001; Kim and Rieke, 2001) with different origins in the retinal circuitry (Sakai et al., 1995; Kim and Rieke, 2001).

Like mean adaptation, contrast adaptation may adjust retinal sensitivity to efficiently accommodate the visual environment (Albrecht et al., 1984). However, the effect of retinal contrast adaptation on visual signals is still poorly understood, particularly in primates. First, although fast adaptation has been documented in primate retina (Benardete et al., 1992; Benardete and Kaplan, 1999), slow adaptation has not, and some reports have suggested that it occurs only in cortex (Maffei et al., 1973; Movshon and Lennie, 1979; Ohzawa et al., 1985; Carandini et al., 1998; Sanchez-Vives et al., 2000a). Second, previous studies in mammalian retina (Shapley and Victor, 1978; 1981; Victor, 1987; Benardete et al., 1992; Brown and Masland, 2001) used linear analysis techniques that did not separate the effect of instantaneous nonlinearities always present in RGC light responses (e.g., spike threshold and saturation) from adaptation (a change in the contrast-response relationship), or used stimulus durations during which slow adaptation was probably altering light responses. Therefore it is unclear how contrast adaptation affects visual sensitivity in steady state. Similarly, it is unclear whether adaptation exerts a homogeneous effect on different types of RGCs, particularly ON and OFF cells, or whether different retinal circuits adapt differently. Finally, it is not known how the sites and mechanisms of adaptation identified in salamander retina (Kim and Rieke, 2001) relate to primate vision because of the dearth of comparative studies.

Using simultaneous recordings from dozens of RGCs, we show that slow contrast adaptation operates in primate retina. To determine how adaptation influences visual signals in steady state, we develop a method to measure the effect of adaptation on sensitivity and kinetics separately from instantaneous nonlineari- 
ties. Sustained increases in contrast significantly attenuated and sped RGC light responses, implying a retinal origin for some of the adaptation observed in psychophysical experiments and in cortex. Adaptation differentially affected response sensitivity and kinetics in ON and OFF RGCs and in subclasses of RGCs, implying that parallel visual pathways adapt differently.

\section{MATERIALS AND METHODS}

Preparation. Eyes were obtained from terminally anesthetized macaque monkeys (Macaca fascicularis, M. mulatta, $M$. radiata) used in other experiments at the Salk Institute and the University of California, San Diego, in accordance with institutional guidelines for the care and use of animals. Immediately after enucleation, the anterior portion of the eye and vitreous were removed in room light, and the eye cup was placed in bicarbonate buffered Ames' solution (Sigma, St. Louis, MO) and stored in darkness for at least $20 \mathrm{~min}$ before dissection. Under infrared illumination, pieces of retina $2-4 \mathrm{~mm}$ in diameter were cut from regions $10-40^{\circ}$ from the fovea and placed flat against a planar array of 61 extracellular microelectrodes that were used to record action potentials from retinal ganglion cells (Meister et al., 1994; Chichilnisky and Baylor, 1999). The preparation was superfused with Ames' solution bubbled with $95 \% \mathrm{O}_{2}$ and $5 \% \mathrm{CO}_{2}$ and maintained at $35-36^{\circ} \mathrm{C}, \mathrm{pH}$ 7.4. In most experiments the piece of retina was separated from the retinal pigment epithelium (RPE) before recording. In 5 of 13 preparations the RPE was left attached. Results from RPE-attached preparations were similar to results from isolated retina preparations.

Larval tiger salamanders (Ambystoma tigrinum) were obtained from Kons Scientific (Germantown, WI) or Charles Sullivan (Nashville, TN). Eyes were removed from dark-adapted $(>2 \mathrm{hr}$ ) salamanders immediately after decapitating and pithing the animal under infrared illumination. The front of the eye was removed, and the eye cup was placed in Ringer's solution containing (in mM): $110 \mathrm{NaCl}, 22 \mathrm{NaHCO}_{3}, 10$ glucose, $2.5 \mathrm{KCl}$, $1.5 \mathrm{CaCl}_{2}, 1.6 \mathrm{MgCl}_{2}$. Pieces of retina $1-1.5 \mathrm{~mm}$ in diameter were isolated from the RPE, mounted on the electrode array for recording as above, and superfused with Ringer's solution bubbled with $95 \% \mathrm{O}_{2}$ and $5 \% \mathrm{CO}_{2}$ and maintained at $21-23^{\circ} \mathrm{C}$ (room temperature), $\mathrm{pH} 7.4$.

Stimuli. The preparation was stimulated with the optically reduced (1.0-1.3 mm diameter) image of a cathode ray tube computer display refreshing at 67 or $120 \mathrm{~Hz}$, focused on the photoreceptor layer by a microscope objective, and centered on the $480 \mu \mathrm{m}$ diameter electrode array. Stimuli were attenuated to low photopic light levels using neutral density filters. In isolated retina experiments the stimulus was delivered from the photoreceptor side. In experiments in which the RPE was attached, the preparation was stimulated from the retinal ganglion cell side through the mostly transparent electrode array. In the latter case the shadows cast by the platinized (black) electrode tips, $5 \mu \mathrm{m}$ in diameter and spaced $60 \mu \mathrm{m}$ apart, had a minimal influence on the intensity or spatial pattern of the stimulus, because they occupied $\sim 1 \%$ of the total area of the array and were optically diffused by virtue of lying in a different focal plane than the photoreceptors.

In monkey experiments, the typical mean photon absorption rate for the long $(\mathrm{L})$, middle $(\mathrm{M})$, and short $(\mathrm{S})$ wavelength sensitive cones was approximately equal to the absorption that would have been caused by spatially uniform monochromatic lights of wavelength 561, 530, and 430 $\mathrm{nm}$ and intensity 8300,8300 , and 4700 photons $\cdot \mu \mathrm{m}^{-2} \cdot \mathrm{sec}^{-1}$, respectively, incident on the photoreceptors (Schnapf et al., 1988). For RPE-attached preparations, this effective intensity included a factor of 2 to account for the light funneling effect of the inner segments (Packer et al., 1996). In some experiments the stimulus was approximately twice or half as intense as the typical value. In salamander experiments, the typical mean photon absorption rate for the L- and S-cones and red rods was approximately equal to the rate that would have been caused by monochromatic lights of wavelength 609,522 , and $441 \mathrm{~nm}$ and intensity 930, 880, and 520 photons $\mu \mathrm{m}^{-2} \cdot \mathrm{sec}^{-1}$, respectively, incident on the photoreceptors (Makino et al., 1991). In one experiment the stimulus was approximately five times as intense, and in some it was $\sim 1.5$ times as intense as the typical value.

The stimulus was a randomly flickering spatially uniform display, with temporal contrast defined as the standard deviation of the intensity divided by the mean. For monkey experiments, random flicker was created by selecting the intensities of the red, green, and blue display guns independently from a Gaussian or binary distribution every $15 \mathrm{msec}$ (67 Hz display) or $8.33 \mathrm{msec}(120 \mathrm{~Hz}$ display). Each continuous run of stimulation at one contrast lasted 5-20 min. This stimulus modulated photon absorptions asynchronously in all three cone types. However, RGCs responded with the same time course and polarity to stimulation by each of the three guns, except for blue-yellow opponent cells (Chichilnisky and Baylor, 1999), which were few and not analyzed. Thus for simplicity only the contrast (modulation of intensity divided by mean) of the green gun, which drove responses most strongly, is plotted in Figures $6,7,8,11$, and 14 . The red and blue gun contributions to light responses were typically $\sim 25$ and $40 \%$ as strong as the green gun contribution, respectively, consistent with a mixture of $\mathrm{L}$ - and $\mathrm{M}$-cone input. No attempt was made to deliver cone isolating stimuli because the overlap in the spectral sensitivity of the L- and M-cones made it difficult to achieve reliable isolation and impossible to achieve contrast $>15 \%$.

For salamander experiments, random flicker stimuli were created by selecting the intensities of the red, green, and blue display guns from a binary (2-valued) distribution every $30 \mathrm{msec}$. Each continuous run of stimulation at one contrast lasted 10-30 min. The gun intensities covaried in fixed ratios chosen to modulate photon absorptions in L-cones [85\% of cones (Sherry et al., 1998)] without modulating absorptions in S-cones ( $8.4 \%$ of cones) or red rods (98\% of rods). The gun intensities required to achieve $\mathrm{L}$-cone isolation were computed using the measured spectral power distributions of each gun (Estevez and Spekreijse, 1982; Wandell, 1995) and the spectral sensitivities of salamander L- and S-cones and red rods (Makino et al., 1991). Because the display had only three primaries, temporal modulation of absorptions in UV cones $(6.8 \%$ of cones) and green rods ( $2 \%$ of rods) could not be avoided. For simplicity the L-cone contrast (modulation of photon absorptions divided by mean) is plotted in Figures 3, 4, 5, 9, and 13.

Recordings. Spike times, peaks, and widths were digitized at a temporal resolution of $0.05 \mathrm{msec}$ (Meister et al., 1994) and stored for off-line analysis. Spikes from 10-50 cells were segregated by manually selecting distinct clusters in scatter plots of spike height and width recorded on each electrode (Meister et al., 1994) and verifying the presence of a refractory period in the spike trains from each cluster. Spikes recorded on multiple electrodes were identified by temporal coincidence; only spikes from the electrode with the most clearly defined cluster were retained. For quantitative analysis of light responses, spike counts from each cell were computed in time bins of length $15 \mathrm{msec}$ ( $67 \mathrm{~Hz}$ display) or $8.33 \mathrm{msec}$ ( $120 \mathrm{~Hz}$ display).

Model of light responses. Retinal ganglion cell light responses display significant nonlinearities (see Figs. $3 B, D, 6 B, D$ ) that render a strictly linear analysis of the effects of contrast adaptation substantially inaccurate (see Figs. $4 C, 7 C$ ). A simple nonlinear model, known as a linearnonlinear ( $\mathrm{LN}$ ) cascade, can capture some of the nonlinearities in light response and thus provide more accurate measurements of contrast adaptation [see Marmarelis and Naka (1972), Korenberg and Hunter (1986), Sakai et al. (1988), and Chichilnisky (2001) for a description of the model and analysis; see Chichilnisky (2001) and Kim and Rieke (2001) for a test of the validity of the model in the present conditions]. In this model the firing rate at each point in time depends only on the value of a generator signal at the same time. The generator signal is assumed to be a linear function, or weighted sum, of recent stimulus modulations. Importantly, the dependence of firing rate on the generator signal may be nonlinear.

The above is not a model of contrast adaptation; however, changes in the parameters of the model obtained with different stimulus contrasts can be used to measure the effects of contrast adaptation. The parameters of the model are the linear filter (weighting of recent stimuli over time) that creates the generator signal and the nonlinearity that transforms the generator signal to firing rate. These parameters were estimated from responses to random flicker stimulation using the procedure described below.

Suppose $M$ is the largest number of time bins over which a stimulus can affect the response. If $s_{\mathrm{t}}$ is a vector of dimension $M$ the entries of which represent stimulus modulation in the $M$ time bins before time $t$, the generator signal $g_{\mathrm{t}}$ at time $t$ is given by $g_{\mathrm{t}}=w \cdot s_{\mathrm{t}}$, where $\cdot$ represents the inner product of vectors and $w$ is a fixed vector of dimension $M$ that represents the linear filter. The firing rate at time $t$ is given by $r_{\mathrm{t}}=N\left(g_{\mathrm{t}}\right)$, where $N$ is an arbitrary real-valued function of its input.

If stimulus intensity in each time bin is drawn from a Gaussian distribution, the linear filter, $w$, can be estimated by computing the spike-triggered average (STA) stimulus defined by 
A

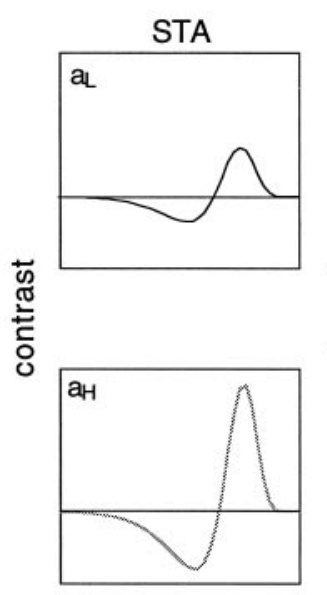

time relative to spike
B

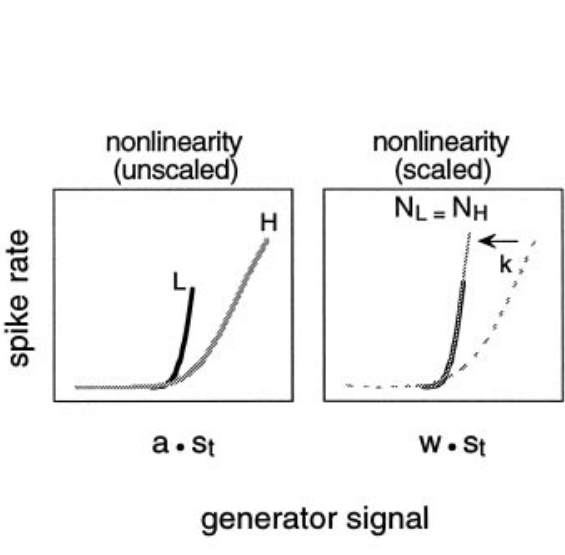

trast linear filter is equal to the low contrast

STA. Thus changes in sensitivity in low and high contrast are referred to changes in the linear filter.

$$
a=\sum_{t=1}^{T} f_{\mathrm{t}} s_{\mathrm{t}} / F,
$$

where $T$ is the number of time bins of the entire recording, $f_{\mathrm{t}}$ is the number of spikes in time bin $t, F$ is the total number of spikes recorded, and $s_{\mathrm{t}}$ is the stimulus immediately preceding time bin $t$. Under the assumptions of the LN model it can be shown that $a$ is directly proportional to $w$ (Chichilnisky, 2001); that is, the STA reveals the shape of the linear filter.

The magnitude of $w$ is indeterminate, however, because the generator signal $g$ has unspecified units. For example, the magnitude of $w$ could be doubled, and the input sensitivity of $N$ halved, without changing the firing rate predictions of the model. Therefore, for simplicity it is assumed that $a=w$ for stimulation at a single contrast. Given this estimate of $w$, to complete the model for the light response required only obtaining an estimate for $N$. The generator signal $g_{\mathrm{t}}$ at each time during stimulation was estimated by summing the elements of the recent stimulus weighted by the linear filter, that is, $g_{\mathrm{t}}=w \cdot s_{\mathrm{t}}$. The spike rate associated with each distinct value of the generator signal was obtained by averaging spike counts over many time points in which nearly the same value of $g$ was observed. This procedure was repeated over the range of observed values of $g$ to determine the relationship between $g$ and average spike rate $r$, that is, the nonlinearity $N$ (see Fig. 3B). To avoid estimation biases, the linear filter and the nonlinearity were estimated using separate segments of recording. This completes the model for light response at one stimulus contrast.

Examination of the effect of contrast adaptation on light responses required determining the magnitude of $w$ in a consistent manner for different stimulus contrasts. This is possible if the form of the associated nonlinearity is unaffected by contrast, in which case any changes in visual processing caused by adaptation are attributable entirely to changes in the linear filter $w$. The procedure for estimating the effects of adaptation is given below and described graphically in Figure 1. The linear filter for low contrast was given by $w_{\mathrm{L}}=a_{\mathrm{L}}$ [the STA in the low contrast condition (Fig. $1 A, D)$ ], and the nonlinear function $N_{\mathrm{L}}$ was obtained as above. The linear filter for high contrast was given by $w_{\mathrm{H}}=k a_{\mathrm{H}}($ Fig. $1 A, D)$, where $k$ was a scale factor selected such that when the nonlinear function $N_{\mathrm{H}}$ for the high contrast condition was computed as above using $w_{\mathrm{H}}$, the functions $N_{\mathrm{H}}$ and $N_{\mathrm{L}}$ superimposed as closely as possible (Fig. $1 C$; see also Figs. $4 D, 7 D$ ), yielding a common nonlinear function $N$. The value of $k$ that yielded closest registration was obtained from parametrized cumulative normal fits to $N_{\mathrm{L}}$ and $N_{\mathrm{H}}$ (Chichilnisky, 2001). Together, $w_{\mathrm{L}}, w_{\mathrm{H}}$, and $N$ provided a combined model of light response for both stimulus contrasts: for high contrast, $g_{\mathrm{t}}=w_{\mathrm{H}} \cdot s_{\mathrm{t}}$, for low contrast $g_{\mathrm{t}}=w_{\mathrm{L}} \cdot s_{\mathrm{t}}$, and for both contrasts $r_{\mathrm{t}}=N\left(g_{\mathrm{t}}\right)$.

Most importantly, changes in light response between the low and high contrast conditions were subsumed entirely by a change in the linear filter from $w_{\mathrm{L}}$ to $w_{\mathrm{H}}$ (Fig. $1 D$ ). For example, if adaptation did not affect light response sensitivity or kinetics, then $w_{\mathrm{L}}$ and $w_{\mathrm{H}}$ would be identical. Alternatively, if sensitivity were reduced during high contrast stimula-
C C D

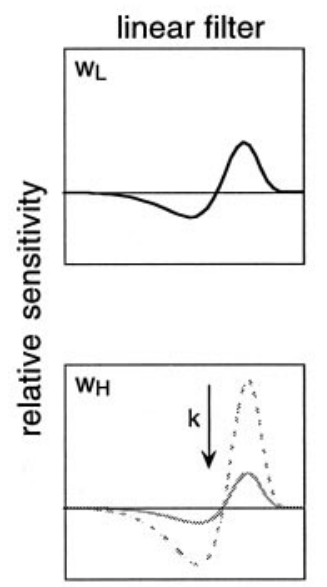

time relative to spike

tion, $w_{\mathrm{H}}$ would have lower amplitude than $w_{\mathrm{L}}$. Note that this procedure also correctly estimates sensitivity in the simpler case of linear light responses. When responses were measured at multiple contrasts, the above procedure was applied to obtain a single nonlinear function $N$ for all contrasts and a distinct linear filter for each contrast (e.g., Figs. 5, 8).

In the case of random flicker generated according to a binary (rather than Gaussian) distribution, the same analysis was applied. In this case $a$ may be approximately proportional to $w$ if the refresh interval of the stimulus is small compared with the integration time of the photoreceptors. In all but one preparation, the refresh interval of binary stimuli was approximately one-third the monkey cone integration time (24 $\mathrm{msec}$ ) (Schnapf et al., 1990) or one-fifth the salamander cone integration time (150 msec) (Matthews et al., 1990). The validity of measurements obtained with binary stimulation was confirmed in two ways. First, in monkey retina, Gaussian (five preparations) and binary (four preparations) stimulation revealed similar changes in sensitivity and kinetics with contrast, and the same pattern of results in ON and OFF cells (see Fig. 12). Second, simulated spike trains created with measured values of $w_{\mathrm{L}}, w_{\mathrm{H}}$, and $N$ and Poisson spike generation, subjected to the analysis procedure above, produced estimates of changes in sensitivity and kinetics that closely paralleled results from real spike trains in both monkey and salamander RGCs. Estimated changes in the peak of the linear filter were typically within $4 \%$ (SD across cells) for simulated and real data; estimated changes in time to zero crossing were within $2 \%$. These estimation errors were uncorrelated across cells and across repeated stimulus presentations, implying significantly lower estimation errors in most of the results presented below. Also, estimation errors were similar in data obtained with Gaussian and binary stimuli.

The linear filters obtained using the above analysis were in some cases compared with those obtained assuming linear light responses (see Figs. $4 C, 7 C)$. In the case of linear light responses, i.e., $r_{\mathrm{t}}=w \cdot s_{\mathrm{t}}$, the leastsquares estimate of $w$ is given by $\bar{r} a / \sigma^{2}$, where $\bar{r}$ is the firing rate during stimulation and $\sigma$ is the standard deviation of the random flicker stimulus (Rieke et al., 1997).

\section{RESULTS}

\section{Slow contrast adaptation in salamander and monkey retina}

Although a fast-onset (tens of milliseconds) form of temporal contrast adaptation has been documented in cat, monkey, and fish retinas (Shapley and Victor, 1978; Victor, 1987; Benardete et al., 1992; Sakai et al., 1995), a slower-onset (tens of seconds) form of adaptation recently described in salamander and rabbit retinas (Smirnakis et al., 1997) has not yet been reported in monkey retina. Figure 2 shows the firing rate of four representative salamander and monkey RGCs as a function of time after the transition from constant full-field illumination to a randomly flickering full-field stimulus. In both species the firing rate de- 
A

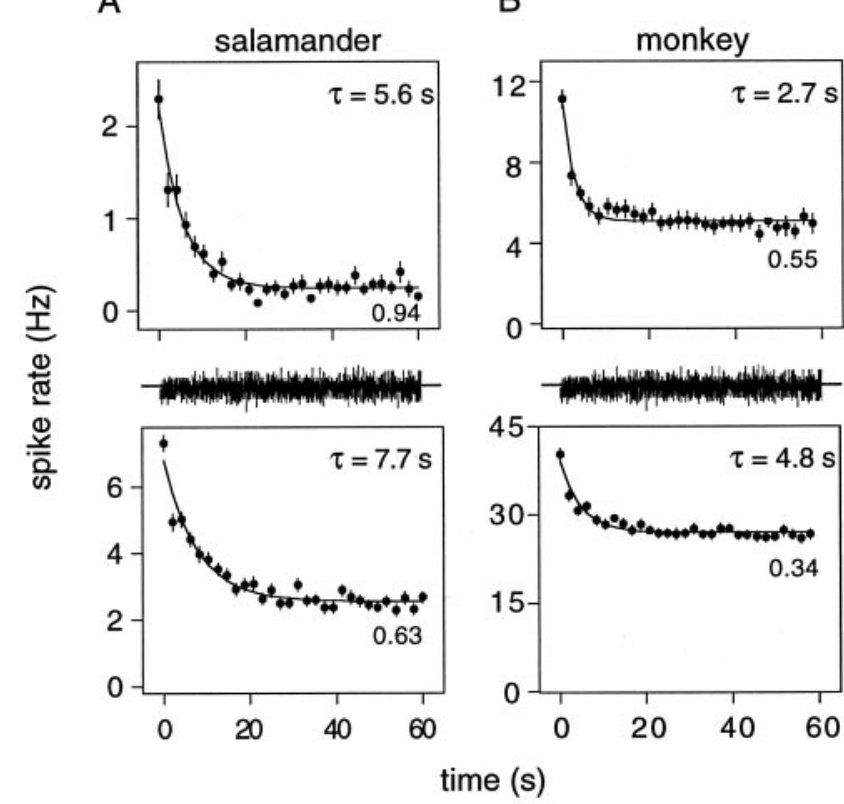

C

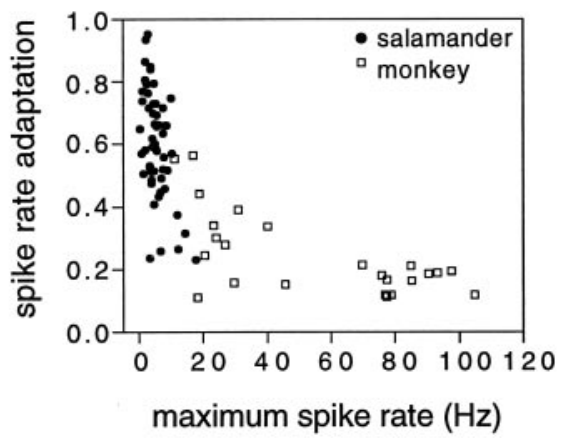

Figure 2. Slow contrast adaptation in salamander and monkey RGCs. Top panels show spike rate as a function of time, averaged over multiple trials in which a randomly flickering stimulus began at time 0 and continued for $60 \mathrm{sec}$. Between trials the retina was exposed to spatially uniform background light of the same mean intensity for approximately $60 \mathrm{sec}$ (data not shown). The random stimulus was different on each trial but had the same time-averaged contrast. $A$, Spike rate is shown as a function of time for two simultaneously recorded salamander RGCs from a single preparation. Stimulus: 60 trials, $33 \mathrm{~Hz}$ binary random flicker, $96 \%$ gun contrast. $B$, Spike rate is shown as a function of time for two simultaneously recorded monkey RGCs from a single preparation. Stimulus: 35 trials, $120 \mathrm{~Hz}$ binary random flicker, $96 \%$ gun contrast. Insets show time constants and fractional reduction in firing rate obtained from exponential fits. Error bars represent \pm 1 SEM. $C$, Fractional reduction in spike rate is shown as a function of maximum spike rate immediately after stimulus onset for cells from four preparations, two salamander (58 cells, ) and two monkey (28 cells, $\square$ ).

cayed from its peak value immediately after stimulus onset to a lower asymptotic value over tens of seconds. The majority of RGCs in salamander (57 of 58 cells, two preparations) and monkey ( 24 of 28 cells, two preparations) displayed a fractional reduction in firing rate $>10 \%$. The mean time constant of adaptation in each preparation obtained from exponential fits ranged from 11 to $16 \mathrm{sec}$ [note that single exponential fits provide only an approximate characterization of the decline in firing rate (Brown and Masland, 2001; Kim and Rieke, 2001)]. The fractional reduction in firing rate was inversely correlated with the peak firing rate at stimulus onset, with a similar trend in salamander and monkey RGCs, as shown in Fig. 2C. These data indicate that similar slow-onset adaptation occurs in salamander and monkey retina. However, from these data alone it is not clear how adaptation affects the sensitivity or kinetics of light responses.

\section{Examining contrast adaptation: characterization of RGC light responses}

To examine how fast and slow contrast adaptation together control visual signals in RGCs, it was desirable to obtain a quantitative characterization of light response that was not confounded by nonlinearities unrelated to adaptation, such as spike threshold and response saturation. Most studies (Shapley and Victor, 1978; Victor, 1987; Benardete et al., 1992; Smirnakis et al., 1997; Brown and Masland, 2001) have relied on linear systems characterizations that can provide incorrect estimates of sensitivity when such nonlinearities are present (see below and Discussion). A minimal extension of the linear model that addresses this problem is a $\mathrm{LN}$ cascade, in which light responses are generated in two stages (for more detail, see Materials and Methods). The first (linear) stage computes the weighted sum of visual inputs over recent time, producing a generator signal. The second (generally nonlinear) stage is a function that determines the firing rate at each point in time based only on the value of the generator signal at the same point in time. One mechanistic interpretation of this model is that retinal circuits sum photoreceptor signals linearly over time to produce synaptic current in RGCs, and spike generation instantaneously and nonlinearly transforms synaptic current to spikes. Although simple, the LN model can also provide an approximation to more complex combinations of linear and nonlinear elements in the retinal circuitry (Kim and Rieke, 2001). The LN model will be used here to describe RGC light responses because (1) it provides a fairly accurate description of responses in the present conditions (Chichilnisky, 2001; Kim and Rieke, 2001) (but see Discussion), and (2) it retains much of the simplicity of a linear model yet can be used to examine contrast adaptation separately from instantaneous response nonlinearities unrelated to adaptation.

Responses to a spatially uniform randomly flickering stimulus were used to obtain direct estimates of the parameters of the LN model, and thus a full characterization of light responses for each cell at each contrast level. The spike-triggered average stimulus (STA) obtained with random flicker revealed how a cell weighted and summed recent stimulus modulations over time. An example is shown in Figure $3 A$. On average, this cell fired after a strong positive modulation preceded by a weaker negative modulation of the stimulus about the mean intensity. If the $\mathrm{LN}$ model is accurate then the STA is proportional to the linear filter that specifies how the cell combined recent stimulus modulations to produce the generator signal. Thus, the stimulus $0-200 \mathrm{msec}$ in the past was weighted positively and strongly, and the stimulus 200-500 msec in the past was weighted negatively and more weakly. Because the dominant, short-latency light sensitivity was positive, this cell was classified as an $\mathrm{ON}$ cell. The cell of Figure $3 C$ responded with opposite polarity and thus was classified as an OFF cell. All recorded cells were unambiguously classified this way.

The model for RGC light responses was completed by obtaining the second stage function that transforms the generator signal into spike rate. This was obtained by plotting the generator signal (stimulus weighted by the linear filter) against the measured spike rate, averaged over many points during recording. Examples are given in Figure $3, B$ and $D$. In both cells the second stage function 
A

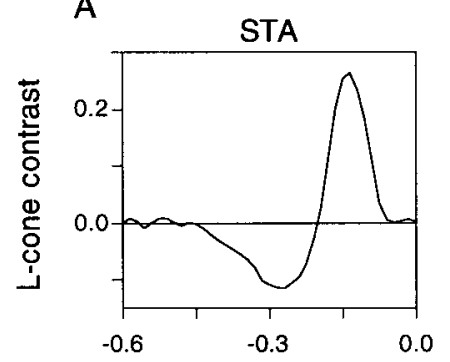

C

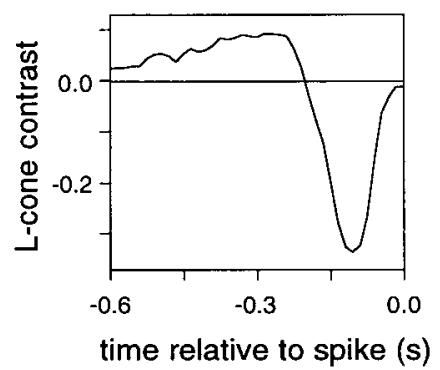

B

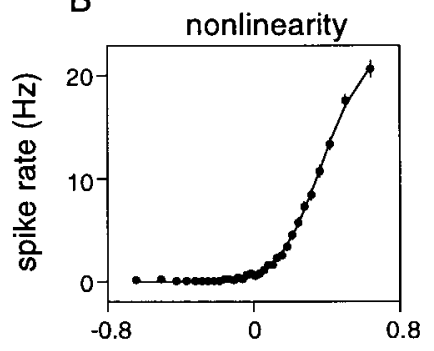

$\mathrm{D}$

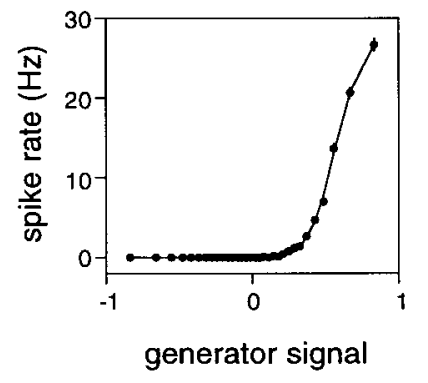

Figure 3. Characterization of light response in one ON cell $(A, B)$ and one OFF cell $(C, D)$ simultaneously recorded in salamander retina. $A, C$, The spike-triggered average $\mathrm{L}$-cone contrast during random flicker stimulation is plotted as a function of time relative to the spike. This is proportional to the linear filtering of recent visual inputs. $B, D$, Spike rate is shown as a function of the estimated generator signal (stimulus weighted by linear filter), averaged over many time points during stimulation. Vertical (horizontal) error bars indicate the SE of spike rate (generator signal) for each such average; most error bars are smaller than the symbols. Smooth curve is a parametrized form of the cumulative normal distribution, shifted and scaled to fit the data. Stimulus: $33 \mathrm{~Hz}$ binary random flicker, $34 \%$ L-cone contrast.

was an accelerating nonlinearity typical of the great majority of RGCs recorded. Some saturation was also evident at high values of the generator signal; this was commonly observed with high contrast stimuli. Were RGC light responses linear in the present conditions, the functions in Figure $3, B$ and $D$, would be linear. The departure from this prediction indicates that the nonlinear second stage of the LN model captured a significant feature of the light response that can create systematic errors in a more restrictive linear analysis of adaptation (see below).

\section{Response nonlinearity in RGCs does not depend on contrast}

To examine temporal contrast adaptation, RGC light responses were characterized as above using random flicker stimuli of low and high contrast. Thus, the stimulus that controlled the state of adaptation was simultaneously used to probe light responses in that state. Because the slowest known component of contrast adaptation operates over tens of seconds (Fig. 2) (Smirnakis et al., 1997; Kim and Rieke, 2001), responses from the first minute of recording (more than three slow adaptation time constants) (Fig. 2) at each contrast level were excluded to confine analysis to the steady state.

Figure $4 A$ shows low and high contrast STAs from a representative OFF cell. The corresponding nonlinearities are shown in Figure $4 B$. Because the units of the generator signal in the $\mathrm{LN}$ model are indeterminate, the linear filter in each condition is known only up to a single, arbitrary scale factor. However, the linear filters obtained with different contrasts can be meaning-

A

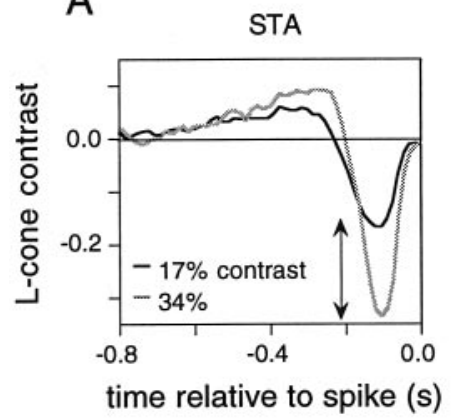

B
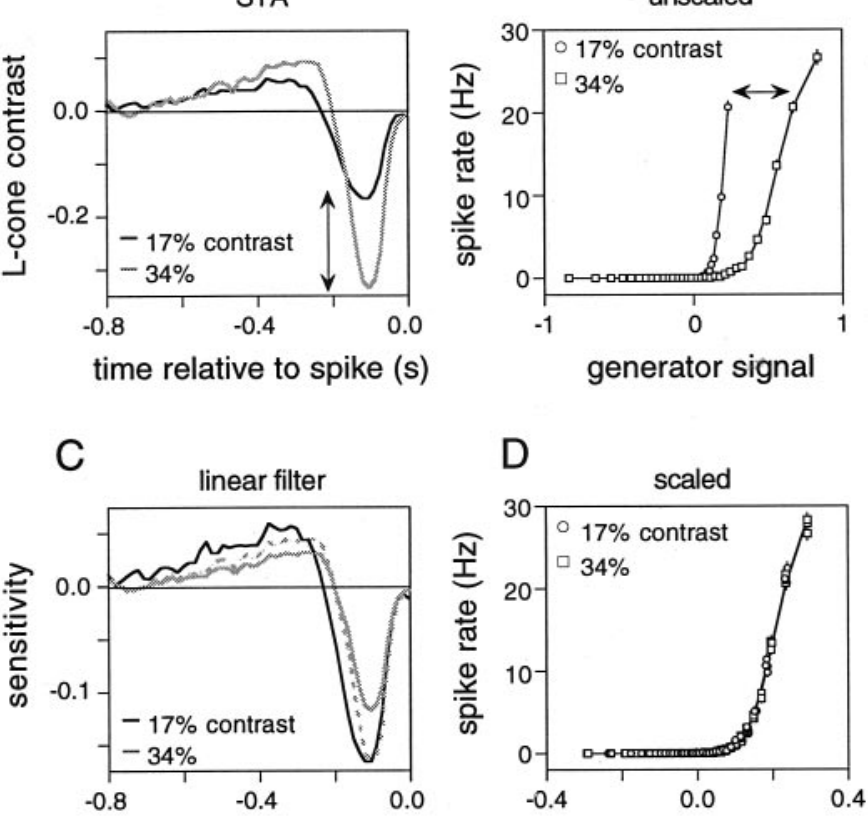

time relative to spike (s)

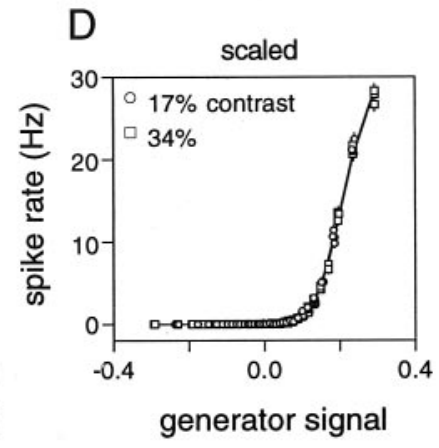

$E$

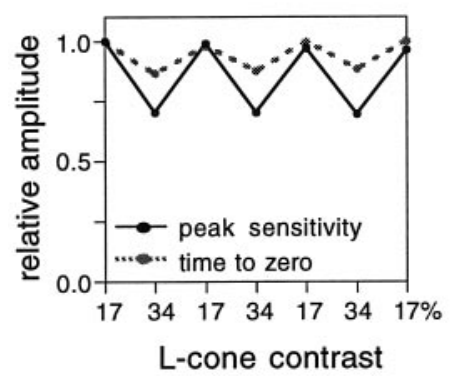

$\mathrm{F}$

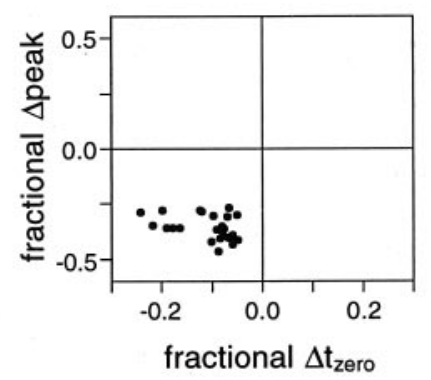

Figure 4. Effect of contrast adaptation on light responses in salamander RGCs. $A$, STAs for a single OFF cell obtained with low $(17 \%$, black trace) and high (34\%, gray trace) contrast stimulation. Stimulus: $33 \mathrm{~Hz}$ $\mathrm{L}$-cone binary random flicker. $B$, Corresponding nonlinearities for low contrast $(\bigcirc)$ and high contrast $(\square)$. Error bars represent \pm 1 SEM (see Fig. 3). $C$, Linear filters: the low contrast STA, and the high contrast STA scaled by 0.35 , are shown with black and gray lines, respectively. The high contrast filter obtained with linear analysis is shown with a dashed gray line. For comparison with the low contrast filter, this was scaled so that its peak divided by the peak of the low contrast filter equals the ratio of the peaks of the high and low contrast filters obtained with linear analysis. $D$, Superimposed nonlinearities from four repeats of low contrast stimulation, and three repeats of high contrast stimulation with abscissa scaled by 0.35. E, Peak sensitivity (solid black) and time to zero crossing (dashed gray) of the linear filter relative to the first low contrast filter for alternating low and high contrast stimulation. $F$, Fractional change in peak sensitivity and time to zero (relative to low contrast) for 24 simultaneously recorded cells including the cell in $A-E$.

fully compared if scaling the amplitude of the high contrast linear filter, and consequently scaling the abscissa of the high contrast nonlinearity, brings the nonlinearities for low and high contrast into register, as is shown in Figure $4 D$ (see Materials and Methods for details). This superposition of nonlinearities in high and low contrast implies that the changes in visual signaling caused by contrast adaptation were attributable to changes in the linear filter, shown in Figure $4 C$. 
A

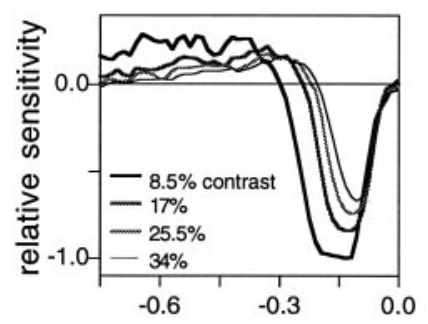

time relative to spike (s)
B

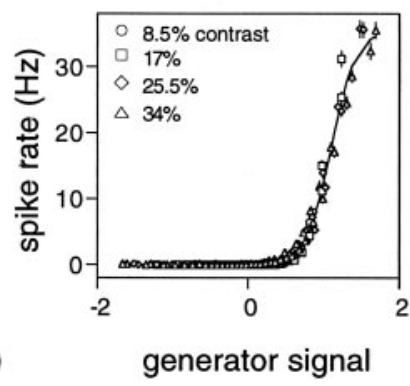

C

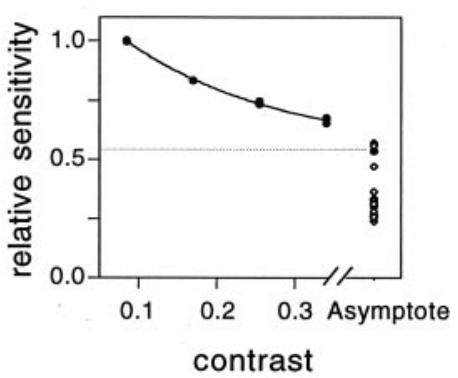

Figure 5. Dependence of sensitivity on contrast. $A$, Linear filters for one salamander RGC at four stimulus contrasts $(8.5,17,25.5$, and $34 \%)$ with decreasing line thickness for higher contrasts; each trace is the average of two stimulus presentations. Stimulus: $33 \mathrm{~Hz}$ $\mathrm{L}$-cone binary random flicker. $B$, Nonlinearities at all four contrasts superimposed. Two repeats at each contrast are shown with separate symbols. Error bars represent \pm 1 SEM (see Fig. 3). $C$, Peak sensitivity (relative to low contrast) as a function of contrast. Two repeats at each contrast are shown with separate symbols. Smooth curve represents an exponential decay to an asymptote of 0.54, shown with a dashed line and filled symbol. Open symbols represent asymptotic peak sensitivity for 15 of 26 other cells recorded in this preparation.

To quantify the registration of the high and low contrast nonlinearities, the correlation coefficient between the measured nonlinearity and a cumulative normal fit (Fig. 4B,D, smooth curves) (Chichilnisky, 2001) was obtained with and without the constraint that the low and high contrast nonlinearities should superimpose. In all but a few of 170 cells recorded in eight preparations, these correlation coefficients were nearly indistinguishable, differing by $<1 \%$ and remaining in the range $0.98-1.0$. Thus, the superposition of nonlinearities in high and low contrast was consistent.

\section{Contrast adaptation alters sensitivity and kinetics of visual signals in RGCs}

The linear filters of Figure $4 C$ indicate $\sim 30 \%$ lower peak sensitivity in high contrast. To confirm that this reduction in sensitivity reflected adaptive visual processing rather than an irreversible decline, the above protocol was performed repeatedly, alternating between low and high contrast. The peak sensitivity in each condition relative to the sensitivity in the first low contrast condition is shown in Figure $4 E$, indicating full reversibility. In what follows, only cells that displayed reversible adaptation were analyzed further.

Accounting for nonlinearities in RGC light responses as above was critical for obtaining accurate estimates of the effects of adaptation on sensitivity: a more standard linear analysis (see Materials and Methods) produced substantially incorrect results. For example, the high contrast filter obtained by assuming linear light responses is shown with a dashed line Figure $4 C$. Taken at face value this would suggest that sensitivity is essentially unchanged by contrast adaptation. Linear analysis usually resulted in substantially incorrect ( $>20 \%$ ) estimates of adaptation (114 of 158 cells). Relative sensitivity in high contrast was sometimes underestimated and frequently overestimated by linear analysis, often resulting in apparently reversed effects of adaptation (57 of 158 cells). These results emphasize the importance of correcting for nonlinearities (see Discussion).

RGC light responses were also sped by adaptation to high contrast stimuli (Shapley and Victor, 1978; Benardete et al., 1992; Smirnakis et al., 1997). This can be observed in Figure $4 C$, where the peak and zero crossing of the linear filter shifted toward the time of the spike in high contrast. This effect, summarized using the time to zero crossing, was also reversible (Fig. $4 E$ ). On average, the time to zero crossing was $13 \%$ lower in high contrast for this cell.

The effects of adaptation were consistent in populations of simultaneously recorded cells. An example is shown in Figure $4 F$, which shows the fractional change in peak sensitivity and the fractional change in the time to zero crossing for 24 simulta- neously recorded cells from the same preparation. Increasing stimulus contrast attenuated and sped the light responses of all cells recorded. Changes in peak sensitivity and time to zero of the light response will be used to describe the effects of contrast adaptation in what follows.

RGC sensitivity declined progressively with stimulus contrast, often approaching a non-zero asymptote at high contrast. Figure 5, $A$ and $B$, shows the linear filters and nonlinearities for a single salamander RGC recorded at four contrasts spanning a fourfold range. As stimulus contrast increased, the amplitude and time to zero of the linear filter progressively decreased. Again, the form of the nonlinearity was essentially unaffected by contrast (Fig. $5 B$ ). Peak sensitivity is shown as a function of contrast in Figure $5 C$. The smooth curve represents an exponential decline to an asymptotic relative sensitivity of 0.54 , apparently representing the limit of adaptation in this cell. In this preparation, an exponential decline adequately described 16 of 27 cells $(50 \%$ lower RMS error than linear fit). For these cells, asymptotic peak sensitivities are shown by the symbols on the right side of Figure $5 C$. This extrapolation suggests that the effect of adaptation on sensitivity is often limited. Similar results were observed in four other preparations.

\section{Contrast adaptation has similar properties in monkey and salamander RGCs}

Contrast adaptation had similar effects on the light responses of monkey RGCs. Figure 6 shows the STAs obtained from two monkey RGCs, one ON and one OFF, at a single contrast, in the same format as Figure 3. The STAs were biphasic, as in salamander, but considerably faster. The relationship between generator signal and spike rate for these cells (Fig. $6 B, D$ ) also resembled results in salamander and indicated significant response nonlinearities typical of most monkey RGCs recorded. Therefore, an examination of sensitivity changes induced by contrast adaptation in monkey RGCs required correcting for response nonlinearities.

Figure 7 shows the effect of contrast adaptation on one monkey OFF RGC, in the same format as Figure 4. Contrast adaptation did not affect the form of the nonlinearity (Fig. $7 B, D$ ); thus the effects of adaptation were attributable to changes in the linear filter: peak sensitivity was lower $(31 \%)$ and kinetics faster $(7 \%)$ in high contrast (Fig. 7C). These effects were reversible (Fig. 7E). Contrast adaptation reduced the peak sensitivity and sped the kinetics of light responses of all cells recorded in this preparation (Fig. $7 F$ ). Note that most of the cells recorded were probably parasol cells (E. J. Chichilnisky and R. S. Kalmar, unpublished observations). 
A

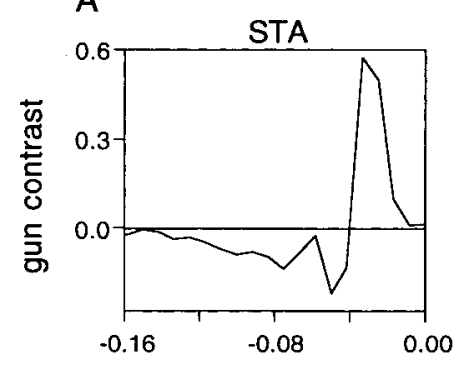

B

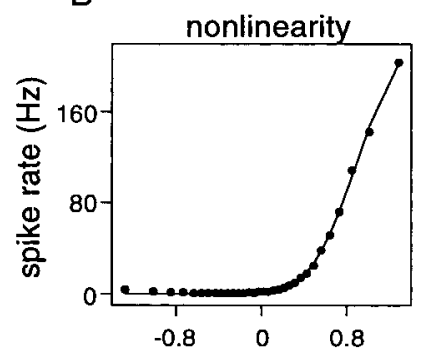

D

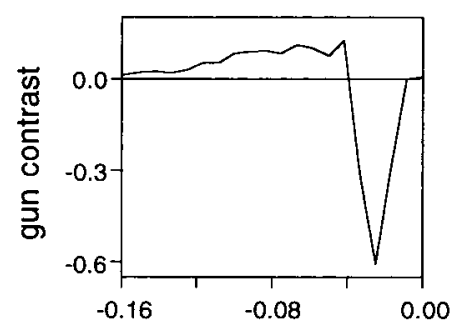

time relative to spike (s)

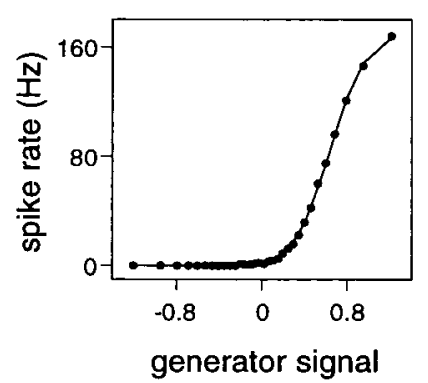

Figure 6. Characterization of light response in one $\mathrm{ON}$ cell $(A, B)$ and one OFF cell $(C, D)$ simultaneously recorded in monkey retina. $A, C$, Spike-triggered average gun contrast during random flicker stimulation as a function of time relative to the spike. $B, D$, Spike rate as a function of the estimated generator signal averaged over many time points during stimulation. Error bars represent \pm 1 SEM (see Fig. 3). Stimulus refresh rate: $120 \mathrm{~Hz}$ binary random flicker, 64\% gun contrast.

As described for salamander RGCs above, the high and low contrast nonlinearities superimposed in all but a few of 194 cells recorded in 13 preparations. Correction for these nonlinearities in light response was critical for obtaining accurate estimates of sensitivity. As in salamander, linear analysis often resulted in substantially incorrect ( $>20 \%$ ) estimates of adaptation (61 of 143 cells). Relative sensitivity in high contrast was sometimes underestimated and frequently overestimated (Fig. $7 C$, dashed line) by linear analysis, often resulting in apparently reversed effects of adaptation ( 24 of 143 cells). These results emphasize the importance of correcting for nonlinearities.

The dependence of sensitivity on temporal contrast also resembled results from salamander retina, as is shown in Figure 8, in the same format as Figure 5. Increases in contrast progressively reduced the peak sensitivity and sped the kinetics of the light response (Fig. $8 A$ ), whereas the form of the nonlinearity was largely unchanged (Fig. $8 B$ ). Figure $8 C$ shows the dependence of sensitivity on contrast and the asymptotic sensitivity from an exponential fit for the same cell. Asymptotic peak sensitivity is also shown for four of nine other simultaneously recorded cells for which sensitivity declined approximately exponentially with contrast (50\% lower RMS error than linear fit). These values were always greater than zero, indicating limited adaptation in many cells. Similar results were observed in four other preparations.

\section{Contrast adaptation affects $\mathrm{ON}$ and OFF RGCs differently}

The above results indicate that contrast adaptation systematically affects steady-state sensitivity and kinetics of RGC light responses and that these features of adaptation are similar in salamander and monkey retina. However, retinal processing is accomplished
A

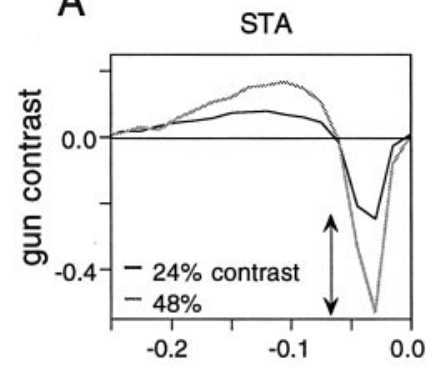

time relative to spike (s)
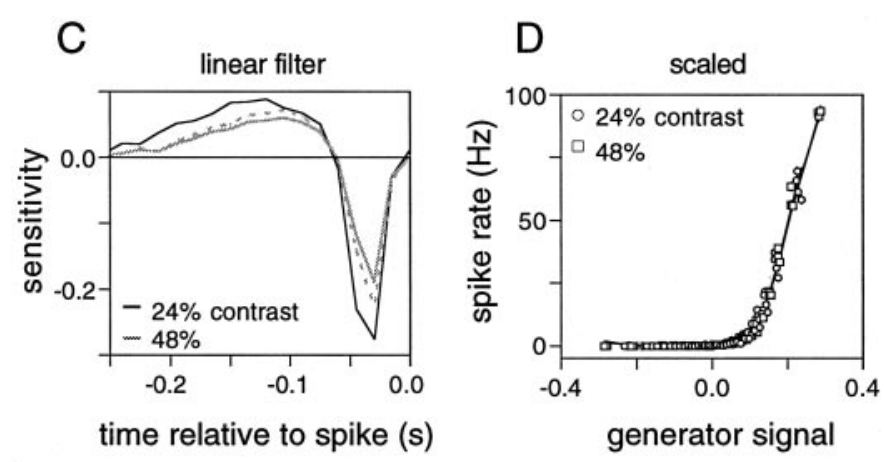

$E$

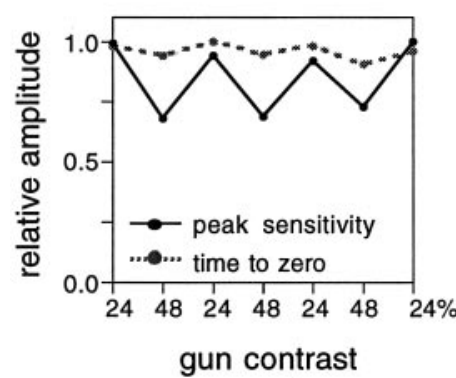

$\mathrm{F}$

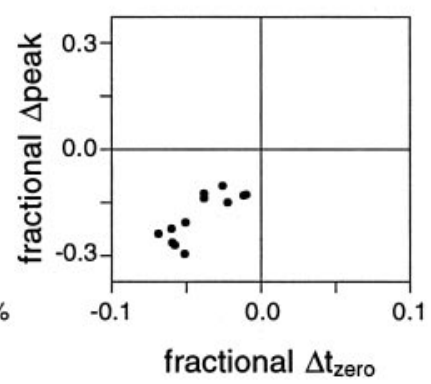

Figure 7. Effect of contrast adaptation on light responses in monkey RGCs. $A$, STAs for a single OFF cell obtained with low $(24 \%$, black trace $)$ and high (48\%, gray trace) contrast stimulation. Stimulus: $67 \mathrm{~Hz}$ Gaussian random flicker. $B$, Corresponding nonlinearities for low contrast $(\bigcirc)$ and high contrast $(\square)$. Error bars represent \pm 1 SEM (see Fig. 3). $C$, Linear filters: the low contrast STA, and the high contrast STA scaled by 0.32 , are shown with black and gray lines, respectively. The high contrast filter obtained with linear analysis is shown with a dashed gray line (see Fig. 4). $D$, Superimposed nonlinearities from four repeats of low contrast stimulation, and three repeats of high contrast stimulation with abscissa scaled by 0.32 . E, Peak sensitivity (solid black) and time to zero crossing (dashed gray) of the linear filter relative to the first low contrast filter for alternating low and high contrast stimulation. $F$, Fractional change in peak sensitivity and time to zero (relative to low contrast) for 12 simultaneously recorded cells including the cell in $A-E$.

by multiple parallel circuits that terminate in morphologically and functionally distinct classes of RGCs with distinct central projections. Is the control of sensitivity homogeneous, or does contrast adaptation differentially affect separate retinal circuits?

This was examined by comparing the effect of contrast on steady-state sensitivity and kinetics of ON and OFF cells. Figure 9 shows results from four representative ON cells and OFF cells simultaneously recorded from one salamander retina. In this preparation, the peak sensitivity of OFF cells was attenuated more by contrast adaptation than that of ON cells ( $p=0.0016 ; 5$ ON cells and 19 OFF cells). 
A

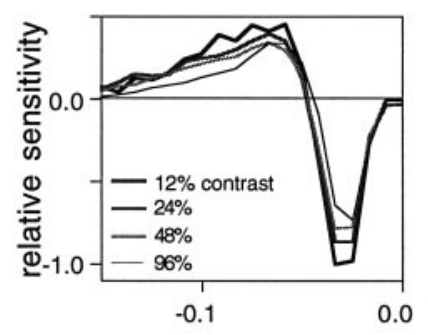

time relative to spike (s)
B

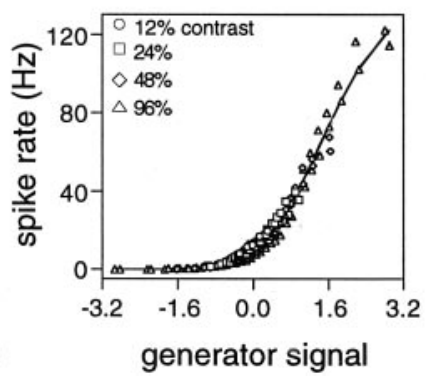

C

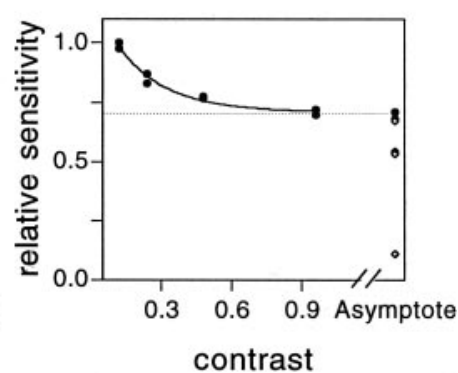

Figure 8. Dependence of sensitivity on contrast. $A$, Linear filters for one monkey RGC at four stimulus contrasts (12, 24,48 , and $96 \%$ ) with decreasing line thickness for higher contrasts; each trace is the average of two stimulus presentations. Stimulus: $120 \mathrm{~Hz}$ binary random flicker. $B$, Nonlinearities at all four contrasts superimposed. Two repeats at each contrast are shown with separate symbols. Error bars represent \pm 1 SEM (see Fig. 3). C, Peak sensitivity (relative to low contrast) as a function of contrast. Two repeats at each contrast are shown with separate symbols. Smooth curve represents an exponential decay to an asymptote of 0.71 , shown with a dashed line and filled symbol. Open symbols represent asymptotic peak sensitivity for four of nine other cells recorded in this preparation.
A
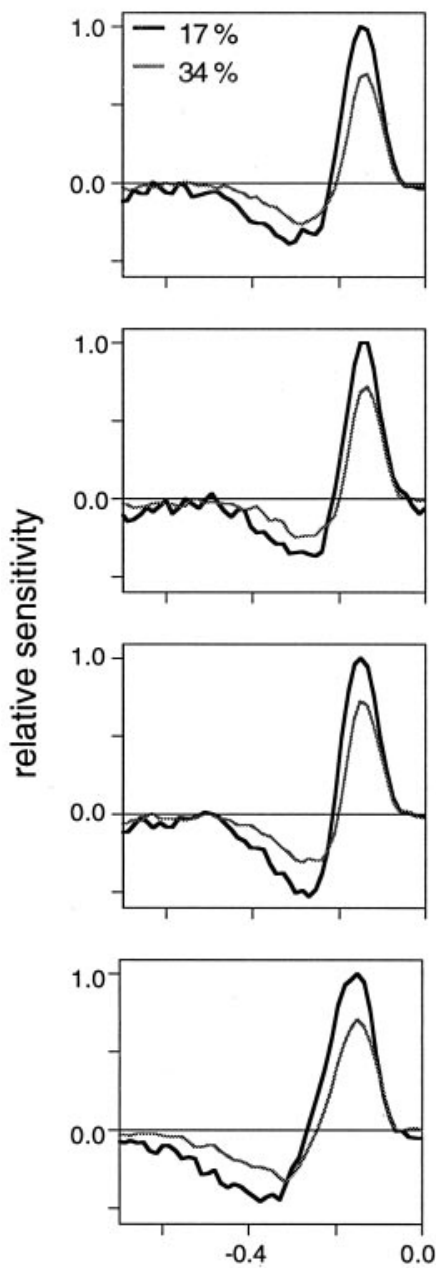

B
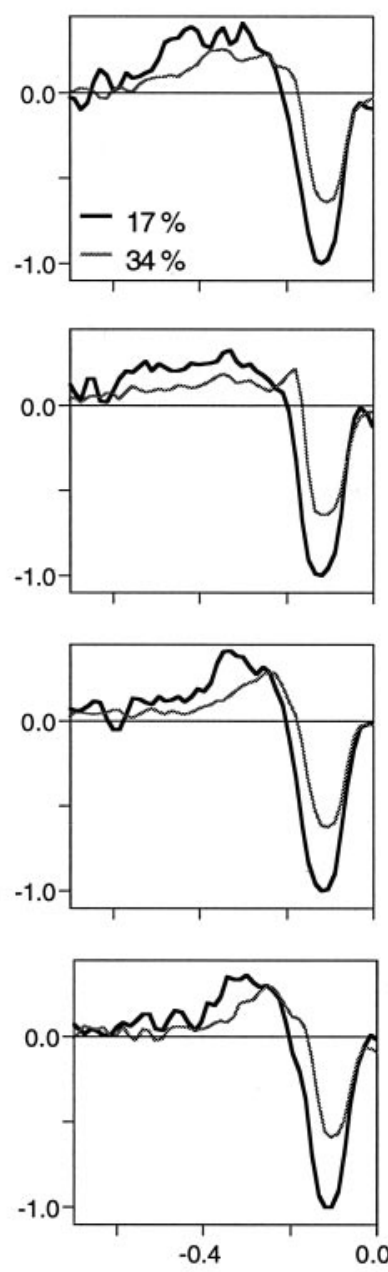

time relative to spike (s)

Figure 9. ON-OFF asymmetry in contrast adaptation, salamander. Linear filters in low (thick, black trace) and high (thin, gray trace) contrast for four ON cells $(A)$ and four OFF cells $(B)$ recorded simultaneously. Stimulus: $33 \mathrm{~Hz}$ binary random flicker, 17 and $34 \%$ L-cone contrast.

This finding was consistent across preparations. Pooled results from eight salamander retinas are shown in Figure $10 \mathrm{~A}$. Each point shows the mean fractional reduction in peak sensitivity in high contrast relative to low contrast for all the ON and OFF cells

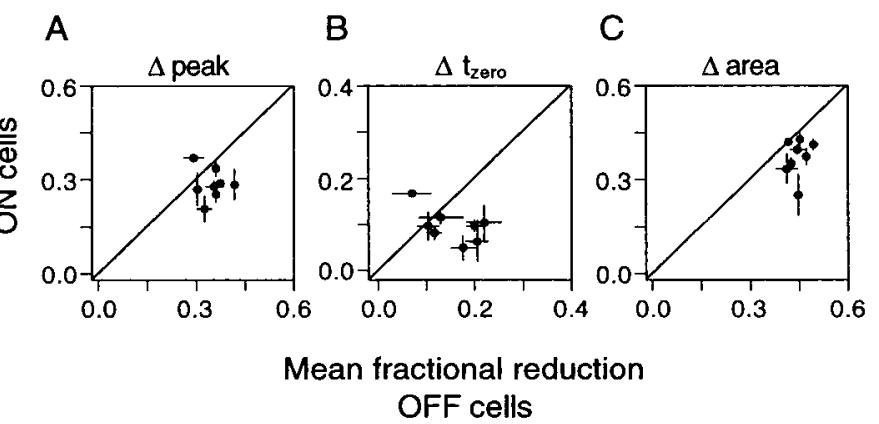

Figure 10. Pooled ON-OFF asymmetry in contrast adaptation, salamander. $A$, Mean fractional reduction in peak sensitivity caused by contrast adaptation for all ON cells and OFF cells in eight preparations; diagonal line represents equality. The dominance of points below the diagonal indicates that in most preparations the mean reduction in sensitivity for OFF cells was greater than that for ON cells. $B$, Mean fractional reduction in time to zero crossing for the same preparations. $C$, Mean fractional reduction in the integrated area under the primary lobe of the linear filter for the same preparations. Each preparation included $3-8 \mathrm{ON}$ cells and 8-29 OFF cells (usually in a 1:3 ratio), for a total of 38 ON cells and 132 OFF cells. Error bars represent \pm 1 SEM.

in one preparation; on average, OFF cells adapted more. Changes in kinetics were also asymmetric. Figure $10 B$ shows the fractional reduction in the time to zero crossing of the light response for the $\mathrm{ON}$ and OFF cells in the same eight salamander retinas. OFF cells showed consistently greater speeding of response kinetics than $\mathrm{ON}$ cells.

The combined effect of a reduction in the peak and time to zero of the linear filter would be expected to reduce the area under the primary lobe of the linear filter, which reflects the integrated strength of the first phase of the visual signal initiated by a brief flash. In accordance with this prediction, asymmetries in the integrated area (Fig. 10C) were more systematic than in the peak sensitivity or time to zero alone. Preparations that showed smaller ON-OFF asymmetries in sensitivity change showed greater asymmetries in kinetic change, and vice versa.

Surprisingly, in monkey retina the asymmetry between $\mathrm{ON}$ and OFF cell sensitivity changes was reversed. This can be seen in Figure 11, which shows the effects of adaptation on four representative ON cells and OFF cells simultaneously recorded from one monkey retina. The peak sensitivity of ON cells was reduced more than that of OFF cells in this preparation $(p<0.0001$; nine $\mathrm{ON}$ and four OFF cells). The same trend is observed in pooled results from eight monkey retinas, shown by the dominance of points above the identity diagonal in Figure $12 \mathrm{~A}$. 
A
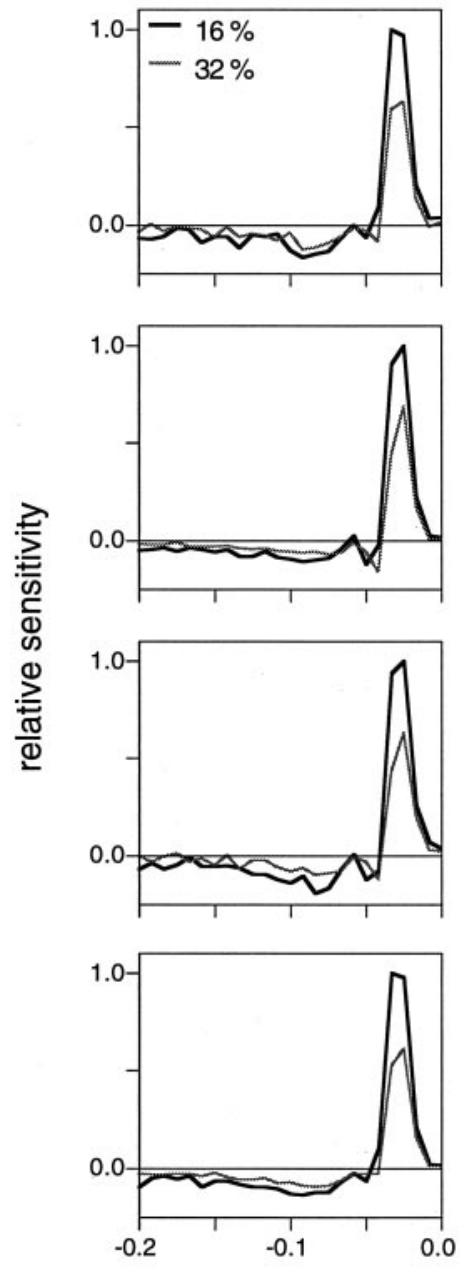

B
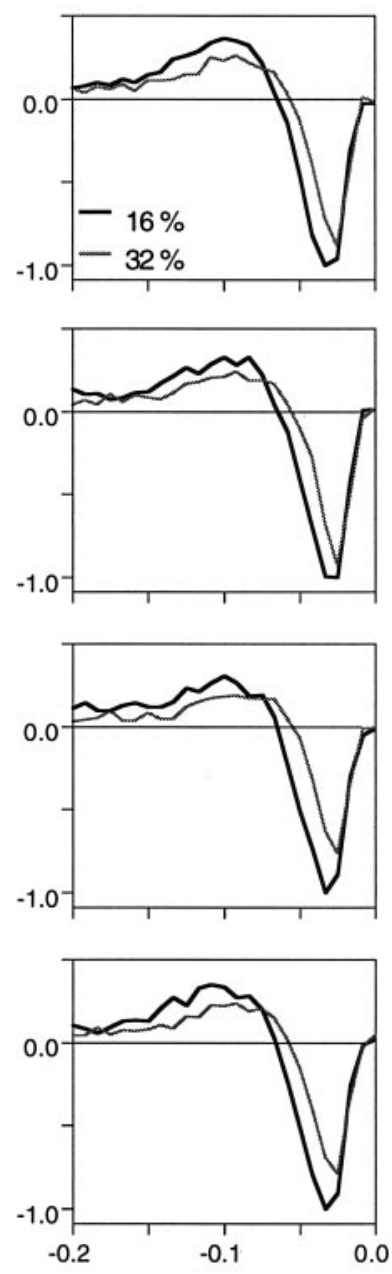

time relative to spike (s)

Figure 11. ON-OFF asymmetry in contrast adaptation, monkey. Linear filters in low (thick, black trace) and high (thin, gray trace) contrast for four ON cells $(A)$ and four OFF cells $(B)$ recorded simultaneously. Stimulus: $120 \mathrm{~Hz}$ binary random flicker, 32 and $64 \%$ gun contrast.

Changes in kinetics of monkey RGCs were also asymmetric, in the same direction as salamander. This is shown for eight preparations in Figure $12 \mathrm{~B}$. OFF cells showed consistently greater change in kinetics than $\mathrm{ON}$ cells. The opposite asymmetries in sensitivity and kinetic adaptation resulted in a less systematic asymmetry in the integrated area under the primary lobe of the linear filter (Fig. 12C) and suggest distinct mechanisms of adaptation in $\mathrm{ON}$ and $\mathrm{OFF}$ retinal circuits.

Note that the extent of contrast adaptation was more variable between preparations in monkey than in salamander. This could have resulted from variation in several experimental parameters, including contrast levels, mean light level, cell types recorded, extent of photopigment bleaching, and retinal eccentricity. Because $\mathrm{ON}-\mathrm{OFF}$ asymmetries were consistent across preparations, these sources of variability were not explored.

\section{Contrast adaptation affects subclasses of RGCs differently}

Multiple anatomically and functionally distinct subtypes of ON and OFF RGCs have long been recognized in many species

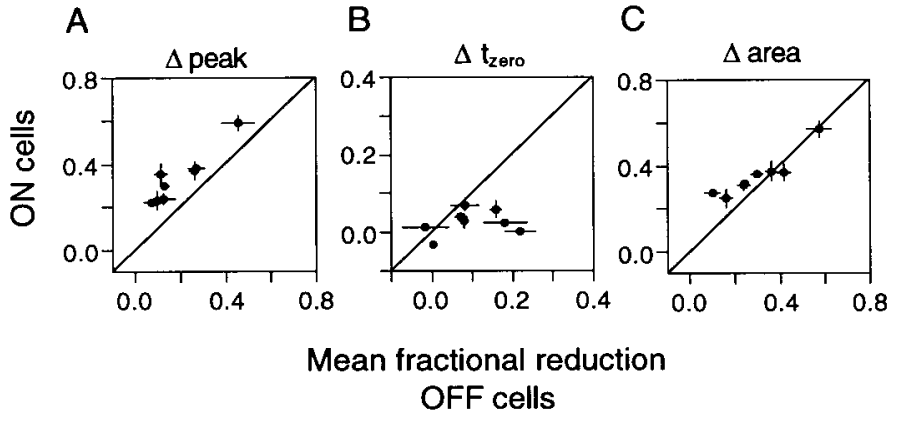

Figure 12. Pooled $\mathrm{ON}-\mathrm{OFF}$ asymmetry in contrast adaptation, monkey. $A$, Mean fractional reduction in peak sensitivity caused by contrast adaptation for all ON cells and OFF cells in eight preparations. $B$, Mean fractional reduction in time to zero crossing for the same preparations. $C$, Mean fractional reduction in the integrated area under the primary lobe of the linear filter for the same preparations. Each preparation included 6-15 ON cells and 3-12 OFF cells, for a total of 76 ON cells and 55 OFF cells. Error bars represent \pm 1 SEM.

(Rodieck, 1998). In the present work, morphological data on the cells recorded was not available, but functionally distinct subclasses of $\mathrm{ON}$ and OFF cells with distinctive and stereotyped light responses were commonly observed in both salamander and monkey retinas. An example is shown in Figure $13 A$, where the time to peak and time to zero crossing of the linear filter is plotted for all OFF cells simultaneously recorded from a single salamander retina. These parameters of the light response fall into distinct clusters that are emphasized with different symbols. The linear filters of cells from two of the clusters $(\square, \boldsymbol{\bullet})$ are shown superimposed for both high and low contrast in Figure 13B. As expected from the parametric clustering, the shapes of the filters were consistent within each group but different between groups.

The effect of adaptation on peak sensitivity is shown for both groups in Figure $13 C$, with each group represented by the same symbols as in Figure 13A. Cells in the first group $(\square)$ showed a systematically greater reduction in sensitivity $(p=0.0014)$ than cells in the second group (-). Results from a different preparation are shown in Figure $13 D-F$. The groups identified in this preparation may or may not correspond to the groups identified in the preparation of Figure $13 A-C$, because they could not be segregated with the same parameters, exhibited different response kinetics, and probably came from a different retinal location. However, functionally distinct classes of cells adapted differently to contrast: the cells indicated by $\square$ showed a larger change in peak sensitivity $(p=0.0005)$ than the cells indicated by $\bullet$. Significant asymmetric adaptation in functional subclasses was observed in six of seven preparations examined.

Similar results were obtained in a subset of monkey retinas. Figure $14 \mathrm{~A}$ shows two subclasses of OFF cells in one monkey retina, identified using the peak and time to zero crossing of the STA, in the same format as Figure $13 A$. The cells indicated by $\square$ (๑) may correspond to midget (parasol) cells; this will be treated in detail elsewhere (Chichilnisky and Kalmar, unpublished observations). Linear filters for the two cell groups, which confirm the parametric clustering, are shown normalized and superimposed in Figure $14 B$.

Figure $14 C$ indicates that the cells represented by $\square$ adapted more strongly $(p=0.024)$. Similar results are shown for two groups of ON cells in Figure $14 D-F(p=0.04)$. Significant asymmetric adaptation in functional subclasses was observed in two of five preparations examined (note that in most cases one subclass consisted of only two cells). 
A

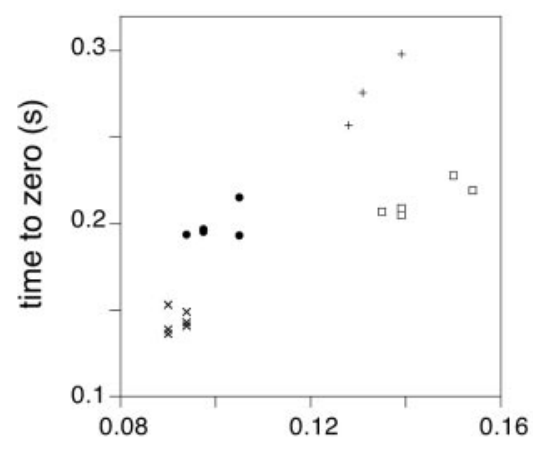

time to peak (s)

D

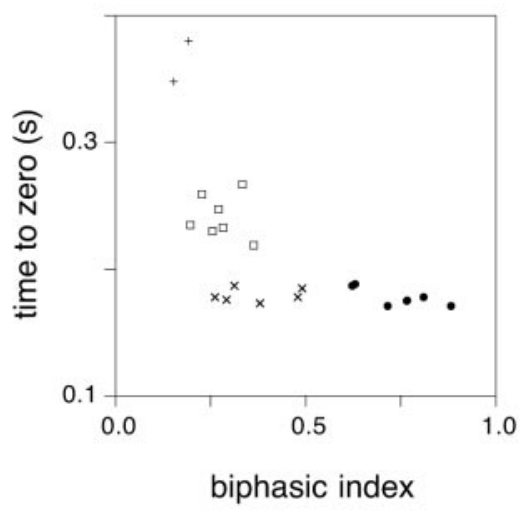

B

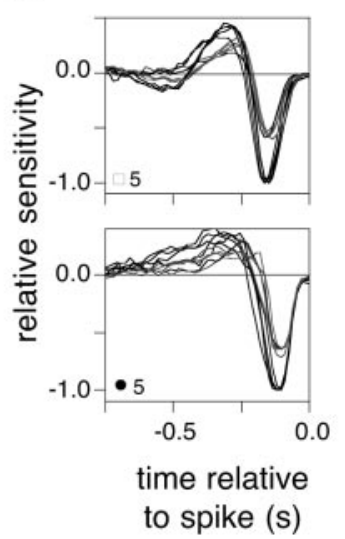

E

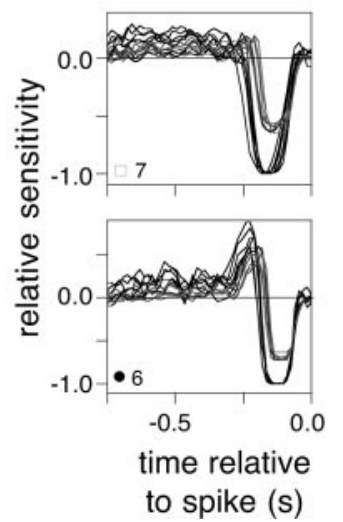

C

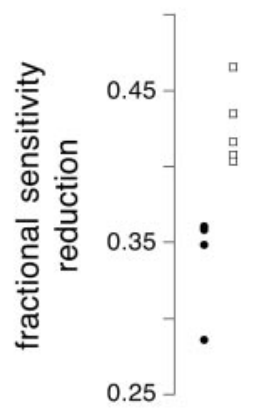

F

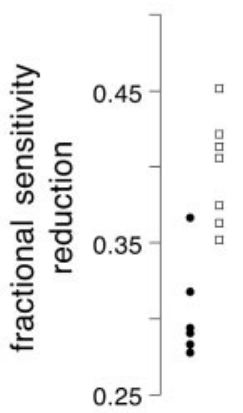

Figure 13. Asymmetric adaptation in subclasses of salamander OFF cells. $A, D$, Each scatter plot shows time to zero crossing versus either time to peak or biphasic index (peak of secondary lobe divided by peak of primary lobe) of the low contrast linear filter for all 19 OFF cells in one preparation $(A)$ and all 21 OFF cells in another preparation $(D)$. Distinct clusters identified by eye were assigned unique symbols. $B, E$, Linear filters for low contrast (black lines) and high contrast (gray lines) of all cells from two of the identified clusters $(\square, 0)$, superimposed and scaled relative to the peak value of each low contrast filter. $C, F$, Fractional reduction in peak sensitivity for cells from both groups, using the same symbols as $A$ and $D$. Stimulus: $33 \mathrm{~Hz}$ binary random flicker, 17 and $34 \%$ L-cone contrast.

\section{DISCUSSION}

This paper contributes five main findings. First, a slow form of temporal contrast adaptation operates in monkey retina, distinct from previously described fast adaptation (contrast gain control) and similar to slow adaptation in salamander. Second, adaptation can be measured separately from instantaneous response nonlinearities unrelated to adaptation that may have confounded previous studies, using a novel analysis. Third, the combined effect of fast and slow adaptation in steady state causes significant and systematic changes in the sensitivity and kinetics of RGC light responses. Therefore contrast adaptation observed in psychophysical experiments and in cortex must originate at least partly in the retina. Fourth, distinct classes of RGCs recorded simultaneously, including ON and OFF cells, adapt differently to contrast. Thus the control of visual sensitivity and kinetics is not homogeneous in parallel visual pathways. Finally, contrast adaptation is similar in salamander and monkey retina, suggesting common mechanisms.

\section{Sites of contrast adaptation in the visual system}

Slow-onset adaptation observed in psychophysical experiments (Blakemore and Campbell, 1969; Lorenceau, 1987; Schieting and Spillmann, 1987; Greenlee et al., 1991; Hammett et al., 1994) and in cortical neurons (Movshon and Lennie, 1979; Albrecht et al., 1984; Ohzawa et al., 1985; Sanchez-Vives et al., 2000a, b) has often been assumed to originate in cortex (Maffei et al., 1973; Movshon and Lennie, 1979; Ohzawa et al., 1985; Carandini et al., 1998; Sanchez-Vives et al., 2000b), because early studies reported little or no adaptation in lateral geniculate nucleus and/or partial interocular transfer (Maffei et al., 1973; Movshon and Lennie,

1979; Ohzawa et al., 1985; Sclar et al., 1985). However, recent studies have reported both fast and slow adaptation in LGN (Sclar, 1987; Shou et al., 1996; Sanchez-Vives et al., 2000a, b; Usrey and Reid, 2000) and that most of the adaptation observed in cortex has monocular origin (Truchard et al., 2000). Indeed, the similar time courses of slow adaptation in retina, cortex, and psychophysical sensitivity suggest they may be related. The present results demonstrate that slow contrast adaptation operates in monkey retina and that in steady state, contrast adaptation strongly and systematically alters response sensitivity and kinetics.

\section{Retinal contrast adaptation: transient versus steady state}

Temporal contrast adaptation consists of at least three kinetic components with distinct origins in the retinal circuitry (Brown and Masland, 2001; Kim and Rieke, 2001). Unfortunately, some previous studies of fast adaptation relied on $30 \mathrm{sec}$ stimulus presentations (Shapley and Victor, 1978; 1981; Victor, 1987; Benardete et al., 1992) during which slower components of adaptation (Fig. 2) (Smirnakis et al., 1997) may have been altering sensitivity. Other studies of slow adaptation in salamander and rabbit retina focused instead on the time course of transient changes in spike rate after abrupt changes in stimulus contrast (Smirnakis et al., 1997; Brown and Masland, 2001); the present results demonstrate similar slow spike rate changes in monkey retina (Fig. 2).

It is not clear from transient rate changes alone, however, how adaptation affects response sensitivity and kinetics in steady state. Analysis of light responses excluding the first minute of recording 
A

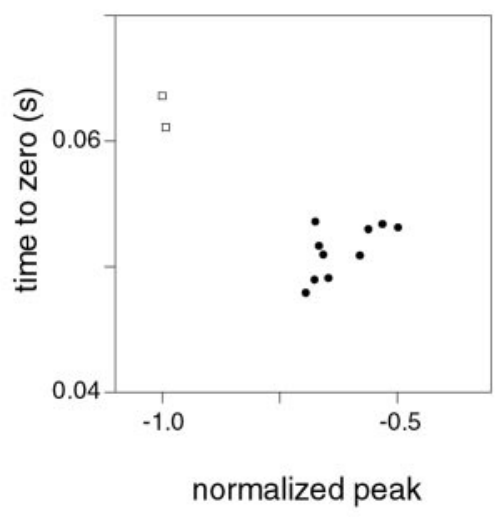

D

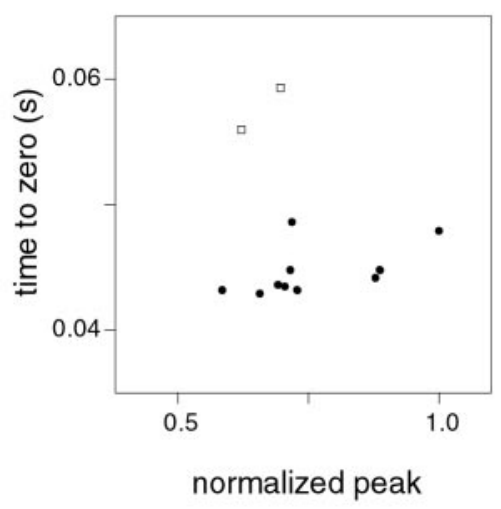

B

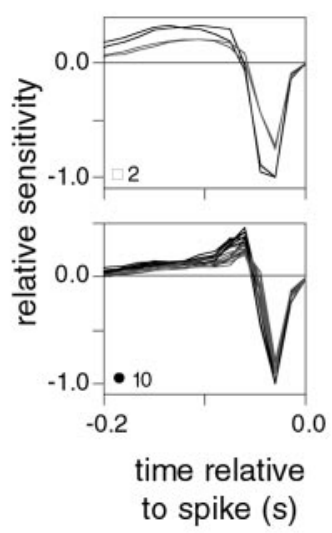

E

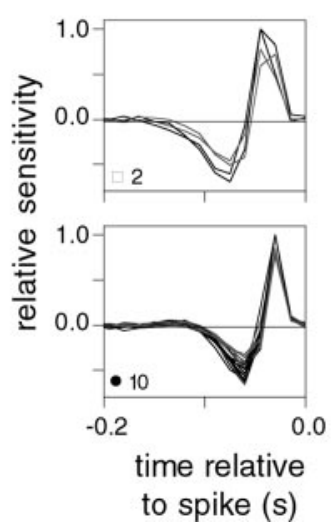

C

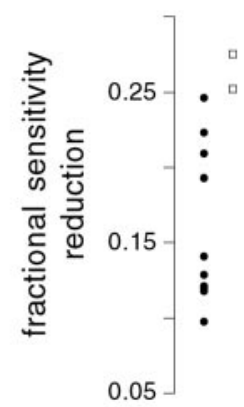

$\mathrm{F}$

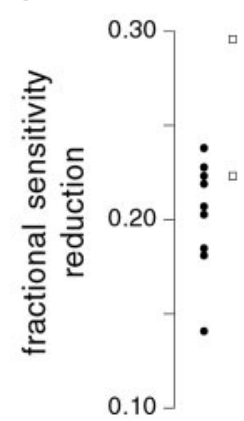

Figure 14. Asymmetric adaptation in subclasses of monkey OFF and ON cells. $A, D$, Each scatter plot shows the time to zero crossing versus the peak of the low contrast linear filter for all 12 OFF cells in one preparation $(A)$ and all 12 ON cells in another preparation $(D)$. Distinct clusters identified by eye were assigned unique symbols. $B, E$, Linear filters for low contrast (black lines) and high contrast (gray lines) of all cells from each of the identified clusters $(\square, \boldsymbol{\bullet})$, superimposed and scaled relative to the peak value of each low contrast filter. $C, F$, Fractional reduction in peak sensitivity for cells from both groups, using the same symbols as $A$ and $D$. Stimulus: $67 \mathrm{~Hz}$ Gaussian random flicker, 12 and $24 \%$ contrast. showed that increases in stimulus contrast systematically reduced RGC sensitivity, as would be expected if the purpose of adaptation were to map the expected range of visual inputs to the finite range of achievable firing rates. Unlike mean adaptation (Shapley and Enroth-Cugell, 1984), contrast adaptation often did not produce steady-state sensitivity inversely proportional to contrast: its effects were more limited (Figs. 5, 8). In steady state, adaptation also altered response kinetics, consistent with previous measurements using several experimental approaches (Shapley and Victor, 1978; 1981; Victor, 1987; Benardete et al., 1992; Kim and Rieke, 2001) (but see Sakai et al., 1995).

\section{Adaptation, response nonlinearities, and the LN model}

Although previous studies have implicated both fast and slow adaptation in RGC light responses, it is difficult to draw firm conclusions from them about the net effects on visual signals. Specifically, studies of fast adaptation (contrast gain control) used essentially linear characterizations of RGC light response at each stimulus contrast (Shapley and Victor, 1978; 1981; Victor, 1987; Benardete et al., 1992), but the present results demonstrate significant nonlinearities in both salamander and monkey RGCs (Figs. 3, 6). These nonlinearities would be expected to contaminate linear analysis of adaptation although they are unrelated to adaptation. For example, response threshold and saturation could cause a $100 \%$ contrast stimulus to produce a response modulation less than twice as large as that produced by a $50 \%$ contrast stimulus and are thus by definition a component of adaptation as analyzed previously. However, response threshold and saturation are fixed limits to stimulus encoding, whereas adaptation implies a change in the input-output relationship to accommodate the visual scene (Shapley and Enroth-Cugell, 1984). These phenomena have different effects on visual signals, may be mediated by different mechanisms, and are perhaps best treated separately. Other studies examined contrast adaptation using random flicker stimulation similar to that used here (Smirnakis et al., 1997; Sakai et al., 1995; Brown and Masland, 2001), but also relied on linear analysis methods.

The present approach was designed to examine adaptation more directly by explicitly measuring an instantaneous response nonlinearity at each contrast and factoring it out (Chander and Chichilnisky, 1999; Baccus and Meister, 2000; Kim and Rieke, 2000, 2001). To accomplish this, the $\mathrm{LN}$ model was used to summarize light responses at each contrast. As with linear models, the LN model cannot account for the dynamics of adaptation, nonlinear feedback (Victor, 1987), or the precise timing of RGC spike trains (Berry et al., 1997; Keat et al., 2001). Indeed, the elements of the LN model probably correspond only loosely to biological mechanisms, and the separation of linear and nonlinear components of light response probably does not reflect isolation of underlying biological processes. Rather, the model provides a tractable summary of light response, with accurate predictions of firing rate over time in response to random flicker stimuli (Chichilnisky, 2001; Kim and Rieke, 2001), and is thus useful for examining the effects of adaptation. Certainly, the model is more accurate than the more restrictive linear models used previously; instantaneous nonlinearities such as thresholding and saturation must contribute to RGC spike trains and are evident in light responses (Figs. 3, 6). Also, linear analysis of sensitivity changes applied to the present data yielded substantially incorrect esti- 
mates of adaptation (Figs. 4C, 7C) and obscured ON-OFF asymmetries (data not shown), perhaps explaining why such asymmetries were not observed in studies that relied on linear analysis (Shapley and Victor, 1978; Benardete et al., 1992; Smirnakis et al., 1997).

\section{Asymmetric adaptation}

The present results demonstrate asymmetric adaptation of sensitivity and kinetics in $\mathrm{ON}$ and OFF RGCs, and in functional subclasses, in salamander and monkey retina. A previous study showed that fast adaptation shifts the temporal frequency tuning of light responses in magnocellular-projecting RGCs but not parvocellular-projecting RGCs of monkey retina (Benardete et al., 1992), paralleling earlier findings in $\mathrm{Y}$ and $\mathrm{X}$ cells in cat retina (Shapley and Victor, 1978). Although these findings implicate light response asymmetries, it is unclear to what extent they reveal asymmetries in steady-state visual sensitivity as opposed to nonlinearities and transient processes (see above). Also, no ONOFF asymmetries were observed. A more recent study (Smirnakis et al., 1997) indicated ON-OFF asymmetries in spike rate adaptation to spatial scale (but not temporal contrast) in salamander RGCs; no asymmetry was observed in rabbit, and sensitivity changes were not examined.

The ON-OFF asymmetries reported here, particularly the opposite sensitivity and kinetic asymmetries in monkey RGCs, suggest different mechanisms of adaptation in the postphotoreceptor retinal circuitry specific to different RGC types. Recent work suggests that some adaptation occurs in bipolar cells (Baccus and Meister, 2000; Kim and Rieke, 2000), where separate $\mathrm{ON}$ and $\mathrm{OFF}$ signals are created. Indeed, in salamander retina the excitatory (likely bipolar) synaptic inputs to OFF RGCs adapt more than inputs to ON RGCs (Kim and Rieke, 2001), similar to the asymmetry reported here. The larger overall gain of OFF synaptic currents in salamander may demand more powerful sensitivity control (Kim and Rieke, 2001). Clearly, this cannot explain the reverse $\mathrm{ON}-\mathrm{OFF}$ asymmetry in monkey retina. In monkey, a weak correlation between receptive field size and sensitivity change was observed (data not shown), and recent findings indicate that $\mathrm{ON}$ cells have larger receptive fields than OFF cells of the same functional type (Chichilnisky and Kalmar, unpublished observations). If adaptation is driven by flux through the receptive field, as has been suggested previously in fast contrast adaptation (Shapley and Victor, 1981) and mean adaptation (Enroth-Cugell and Shapley, 1973), stronger adaptation in ON cells might be expected.

Note that because the dependence of sensitivity on contrast (Figs. 5C, 8C) sometimes differed in ON and OFF cell subpopulations (data not shown), the ON-OFF asymmetries reported here could in principle be reversed for certain contrast pairs, although this was not observed. Also, if mean and contrast adaptation interact, the direction of the ON-OFF asymmetry could vary with light level.

A consequence of asymmetric adaptation in the ON and OFF pathways is that they cannot be considered mirror-symmetric systems, or asymmetric systems in a fixed relationship. Psychophysical experiments have shown that adaptation to uniform background light exerts asymmetric effects on the appearance of increments and decrements (Chichilnisky and Wandell, 1996). Such perceptual phenomena could reflect asymmetric adaptation in the ON and OFF pathways.

\section{REFERENCES}

Albrecht DG, Farrar SB, Hamilton DB (1984) Spatial contrast adaptation characteristics of neurones recorded in the cat's visual cortex. J Physiol (Lond) 347:713-739.

Anstis S (1996) Adaptation to peripheral flicker. Vision Res 36:3479-3485.

Baccus S, Meister M (2000) Mechanisms of contrast adaptation in the retina. Soc Neurosci Abstr 26:1328.

Benardete EA, Kaplan E (1999) The dynamics of primate m retinal ganglion cells. Vis Neurosci 16:355-368.

Benardete EA, Kaplan E, Knight BW (1992) Contrast gain control in the primate retina: $\mathrm{P}$ cells are not $\mathrm{X}$-like, some $\mathrm{M}$ cells are. Vis Neurosci 8:483-486.

Berry MJ, Warland DK, Meister M (1997) The structure and precision of retinal spike trains. Proc Natl Acad Sci USA 94:5411-5416.

Blakemore C, Campbell FW (1969) On the existence of neurones in the human visual system selectively sensitive to the orientation and size of retinal images. J Physiol (Lond) 203:237-260.

Brown SP, Masland RH (2001) Spatial scale and cellular substrate of contrast adaptation by retinal ganglion cells. Nat Neurosci 4:44-51.

Carandini M, Movshon JA, Ferster D (1998) Pattern adaptation and cross-orientation interactions in the primary visual cortex. Neuropharmacology 37:501-511.

Chander D, Chichilnisky EJ (1999) Contrast adaptation and gain changes in salamander and monkey retina. Soc Neurosci Abstr 25:1431.

Chichilnisky EJ (2001) A simple white noise analysis of neuronal light responses. Network: Comput Neural Syst 12:199-213.

Chichilnisky EJ, Baylor DA (1999) Receptive-field microstructure of blue-yellow ganglion cells in primate retina. Nat Neurosci 2:889-893.

Chichilnisky EJ, Wandell BA (1996) Seeing gray through the ON and OFF pathways. Vis Neurosci 13:591-596.

Enroth-Cugell C, Shapley RM (1973) Flux, not retinal illumination, is what cat retinal ganglion cells really care about. J Physiol (Lond) 233:311-326.

Estevez O, Spekreijse H (1982) The silent substitution method in visual research. Vision Res 22:681-691.

Greenlee MW, Georgeson MA, Magnussen S, Harris JP (1991) The time course of adaptation to spatial contrast. Vision Res 31:223-236.

Hammett ST, Snowden RJ, Smith AT (1994) Perceived contrast as a function of adaptation duration. Vision Res 34:31-40.

Keat J, Reinagel P, Reid RC, Meister M (2001) Predicting every spike: a model for the responses of visual neurons. Neuron 30:803-817.

Kim KJ, Rieke F (2000) Temporal contrast adaptation in the salamander retina. Invest Ophthalmol Vis Sci [Suppl] 41:937.

Kim KJ, Rieke F (2001) Temporal contrast adaptation in the input and output signals of salamander retinal ganglion cells. J Neurosci 21:287-299

Korenberg MJ, Hunter IW (1986) The identification of nonlinear biological systems: LNL cascade models. Biol Cybern 55:125-134.

Lorenceau J (1987) Recovery from contrast adaptation: effects of spatial and temporal frequency. Vision Res 27:2185-2191.

Maffei L, Fiorentini A, Bisti S (1973) Neural correlate of perceptual adaptation to gratings. Science 182:1036-1038.

Makino CL, Taylor WR, Baylor DA (1991) Rapid charge movements and photosensitivity of visual pigments in salamander rods and cones. J Physiol (Lond) 442:761-780.

Marmarelis PZ, Naka K (1972) White-noise analysis of a neuron chain: an application of the Wiener theory. Science 175:1276-1278.

Matthews HR, Fain GL, Murphy RL, Lamb TD (1990) Light adaptation in cone photoreceptors of the salamander: a role for cytoplasmic calcium. J Physiol (Lond) 420:447-469.

Meister M, Pine J, Baylor DA (1994) Multi-neuronal signals from the retina: acquisition and analysis. J Neurosci Methods 51:95-106.

Movshon JA, Lennie P (1979) Pattern-selective adaptation in visual cortical neurones. Nature 278:850-852.

Ohzawa I, Sclar G, Freeman RD (1985) Contrast gain control in the cat's visual system. J Neurophysiol 54:651-667.

Packer OS, Williams DR, Bensinger DG (1996) Photopigment transmittance imaging of the primate photoreceptor mosaic. J Neurosci 16:2251-2260.

Rieke FM, Warland D, Steveninck RR, Bialek W (1997) Spikes: exploring the neural code. Cambridge, MA: MIT.

Rodieck RW (1998) The first steps in seeing. Sunderland, MA: Sinauer.

Sakai HM, Wang JL, Naka K (1995) Contrast gain control in the lower vertebrate retinas. J Gen Physiol 105:815-835.

Sakai HM, Naka K, Korenberg MJ (1988) White-noise analysis in visual neuroscience. Vis Neurosci 1:287-296.

Sanchez-Vives MV, Nowak LG, McCormick DA (2000a) Membrane mechanisms underlying contrast adaptation in cat area 17 in vivo. J Neurosci 20:4267-4285.

Sanchez-Vives M V, Nowak LG, McCormick DA (2000b) Cellular mechanisms of long-lasting adaptation in visual cortical neurons in vitro. J Neurosci 20:4286-4299. 
Schieting S, Spillmann L (1987) Flicker adaptation in the peripheral retina. Vision Res 27:277-284.

Schnapf JL, Kraft TW, Nunn BJ, Baylor DA (1988) Spectral sensitivity of primate photoreceptors. Vis Neurosci 1:255-261.

Schnapf JL, Nunn BJ, Meister M, Baylor DA (1990) Visual transduction in cones of the monkey Macaca fascicularis. J Physiol (Lond) 427:681713.

Sclar G (1987) Expression of "retinal" contrast gain control by neurons of the cat's lateral geniculate nucleus. Exp Brain Res 66:589-596.

Sclar G, Ohzawa I, Freeman RD (1985) Contrast gain control in the kitten's visual system. J Neurophysiol 54:668-675.

Shapley RM, Enroth-Cugell C (1984) Visual adaptation and retinal gain controls. Prog Retinal Res 3:263-346.

Shapley RM, Victor JD (1978) The effect of contrast on the transfer properties of cat retinal ganglion cells. J Physiol (Lond) 285:275-298.

Shapley RM, Victor JD (1981) How the contrast gain control modifies the frequency responses of cat retinal ganglion cells. J Physiol (Lond) 318:161-179.

Sherry DM, Bui DD, Degrip WJ (1998) Identification and distribution of photoreceptor subtypes in the neotenic tiger salamander retina. Vis Neurosci 15:1175-1187.

Shou T, Li X, Zhou Y, Hu B (1996) Adaptation of visually evoked responses of relay cells in the dorsal lateral geniculate nucleus of the cat following prolonged exposure to drifting gratings. Vis Neurosci 13:605-613.

Smirnakis SM, Berry MJ, Warland DK, Bialek W, Meister M (1997) Adaptation of retinal processing to image contrast and spatial scale. Nature 386:69-73.

Truchard AM, Ohzawa I, Freeman RD (2000) Contrast gain control in the visual cortex: monocular versus binocular mechanisms. J Neurosci 20:3017-3032.

Usrey WM, Reid RC (2000) Visual physiology of the lateral geniculate nucleus in two species of new world monkey: Saimiri sciureus and Aotus trivirgatis. J Physiol (Lond) 523:755-769.

Victor JD (1987) The dynamics of the cat retinal X cell centre. J Physiol (Lond) 386:219-246.

Wandell BA (1995) Foundations of vision. Sunderland, MA: Sinauer. 